\title{
Correction: Candidemia Risk Prediction (CanDETEC) Model for Patients With Malignancy: Model Development and Validation in a Single-Center Retrospective Study
}

Junsang Yoo ${ }^{1 *}$, RN, PhD; Si-Ho Kim²*, MD; Sujeong Hur ${ }^{3,4}$, RN, MS; Juhyung Ha ${ }^{5}$; Kyungmin $\mathrm{Huh}^{6}$, MD; Won Chul Cha ${ }^{4,7,8}, \mathrm{MD}$

${ }^{1}$ Department of Nursing, College of Nursing, Sahmyook University, Seoul, Republic of Korea

${ }^{2}$ Division of Infectious Disease, Samsung Changwon Hospital, Sungkyunkwan University School of Medicine, Changwon, Republic of Korea

${ }^{3}$ Department of Patient Experience Management, Samsung Medical Center, Seoul, Republic of Korea

${ }^{4}$ Department of Digital Health, Samsung Advanced Institute for Health Sciences \& Technology, Sungkyunkwan University, Seoul, Republic of Korea

${ }^{5}$ Department of Computer Science, Indiana University Bloomington, Bloomington, IN, United States

${ }^{6}$ Division of Infectious Disease, Samsung Medical Center, Sungkyunkwan University School of Medicine, Seoul, Republic of Korea

${ }^{7}$ Department of Emergency Medicine, Samsung Medical Center, Sungkyunkwan University School of Medicine, Seoul, Republic of Korea

${ }^{8}$ Digital Innovation Center, Samsung Medical Center, Seoul, Republic of Korea

* these authors contributed equally

\section{Corresponding Author:}

Won Chul Cha, MD

Department of Emergency Medicine

Samsung Medical Center

Sungkyunkwan University School of Medicine

81, Irwon-ro, Gangnam-gu

Seoul, 06351

Republic of Korea

Phone: 82234102053

Email: wc.cha@samsung.com

\section{Related Article:}

Correction of: https://medinform.jmir.org/2021/7/e24651

(JMIR Med Inform 2022;10(1):e36385) doi: 10.2196/36385

In "Candidemia Risk Prediction (CanDETEC) Model for Patients With Malignancy: Model Development and Validation in a Single-Center Retrospective Study" (JMIR Med Inform 2021;9(7):e24651), one error was noted.

Due to a system error, the ORCID number of author Sujeong Hur was incorrectly published as:

0000-0001-7763-8940
This has been corrected to:

0000-0003-1335-576X

The correction will appear in the online version of the paper on the JMIR Publications website on January 19, 2022, together with the publication of this correction notice. Because this was made after submission to PubMed, PubMed Central, and other full-text repositories, the corrected article has also been resubmitted to those repositories.

This is a non-peer-reviewed article. Submitted 12.01.22; accepted 12.01.22; published 19.01.22.

Please cite as:

Yoo J, Kim SH, Hur S, Ha J, Huh K, Cha WC

Correction: Candidemia Risk Prediction (CanDETEC) Model for Patients With Malignancy: Model Development and Validation in a Single-Center Retrospective Study

JMIR Med Inform 2022;10(1):e36385

URL: https://medinform.jmir.org/2022/1/e36385

doi: $\underline{10.2196 / 36385}$

PMID: 
CJunsang Yoo, Si-Ho Kim, Sujeong Hur, Juhyung Ha, Kyungmin Huh, Won Chul Cha. Originally published in JMIR Medical Informatics (https://medinform.jmir.org), 19.01.2022. This is an open-access article distributed under the terms of the Creative Commons Attribution License (https://creativecommons.org/licenses/by/4.0/), which permits unrestricted use, distribution, and reproduction in any medium, provided the original work, first published in JMIR Medical Informatics, is properly cited. The complete bibliographic information, a link to the original publication on https://medinform.jmir.org/, as well as this copyright and license information must be included. 